\title{
Durvalumab-associated Late-onset Myocarditis Successfully Treated with Corticosteroid Therapy
}

\author{
Tomoki Maetani ${ }^{1}$, Toka Hamaguchi ${ }^{2}$, Takafumi Nishimura ${ }^{3}$, \\ Satoshi Marumo ${ }^{1}$ and Motonari Fukui ${ }^{1}$
}

\begin{abstract}
:
We herein report a 66-year-old man with locally advanced non-small-cell lung cancer (NSCLC) who developed durvalumab-associated myocarditis. The patient underwent durvalumab administration every two weeks following concurrent chemoradiotherapy (CCRT), without any adverse events or apparent disease progression. He presented with fatigue and dyspnea on exertion seven months after the first administration. Myocarditis was suspected based on laboratory data, an electrocardiogram, echocardiography, and magnetic resonance imaging findings. The definitive diagnosis was confirmed by a myocardial biopsy. Myocarditis was alleviated by cessation of durvalumab and corticosteroid therapy. This is a noteworthy case to describe late-onset myocarditis following the administration of durvalumab for NSCLC.
\end{abstract}

Key words: durvalumab, lung cancer, irAE, myocarditis

(Intern Med Advance Publication)

(DOI: 10.2169/internalmedicine.7644-21)

\section{Introduction}

Immune checkpoint inhibitors (ICIs) targeting programmed cell death 1 (PD-1) or programmed cell death ligand 1 (PD-L1) are now widely used in the treatment of lung cancer. Consolidation therapy with durvalumab, a monoclonal antibody against PD-L1, has been shown to improve the outcome of definitive concurrent chemoradiotherapy (CCRT) for locally advanced non-small-cell lung cancer (NSCLC) (1). However, ICIs may induce harmful adverse events related to the immune reaction, called immune-related adverse events (irAEs) (2).

Several case reports and case series have documented myocarditis with concomitant ICIs (3-5). Although a case of myocarditis after tremelimumab plus durvalumab for endometrial cancer has been reported (6), to our knowledge, there are currently no detailed case reports about myocarditis following the administration of durvalumab for NSCLC aside from the one presented here. Furthermore, the present case is notable for its late onset.

\section{Case Report}

A 66-year-old man who was a former smoker presented with a 2-day history of fatigue and dyspnea on exertion. Nine months earlier, he had been diagnosed with stage IIB NSCLC (adenocarcinoma). At the time of the diagnosis of NSCLC, he had a primary lesion $35 \times 18 \mathrm{~mm}$ in size on the right upper lobe of his lung and right hilar lymph node metastasis.

Radical surgical resection was not recommended due to large hilar lymph node metastasis and invasion to the right pulmonary artery. He therefore underwent CCRT, including carboplatin and paclitaxel, and external beam radiotherapy. Radiotherapy was delivered to the primary lesion, involved hilar lymph node, and adjacent mediastinum, using 3dimensional conformal radiotherapy for a total dose of 60 Gy in 30 fractions (2 Gy per fraction once daily). The heart was outside the irradiation field (Fig. 1). After CCRT, he was administered durvalumab at $10 \mathrm{mg} / \mathrm{kg}$ every 2 weeks for 14 courses over 7 months. He had hypertension and a history of abdominal aortic aneurysm rupture treated with

${ }^{1}$ Tazuke Kofukai Medical Research Institute, Respiratory Disease Center, Japan, ${ }^{2}$ Tazuke Kofukai Medical Research Institute, Cardiovascular Center, Japan and ${ }^{3}$ Department of Medical Oncology, Tazuke Kofukai Medical Research Institute, Japan Received: March 31, 2021; Accepted: July 2, 2021; Advance Publication by J-STAGE: August 24, 2021 Correspondence to Dr. Tomoki Maetani, tomoki.maetani1991@gmail.com 


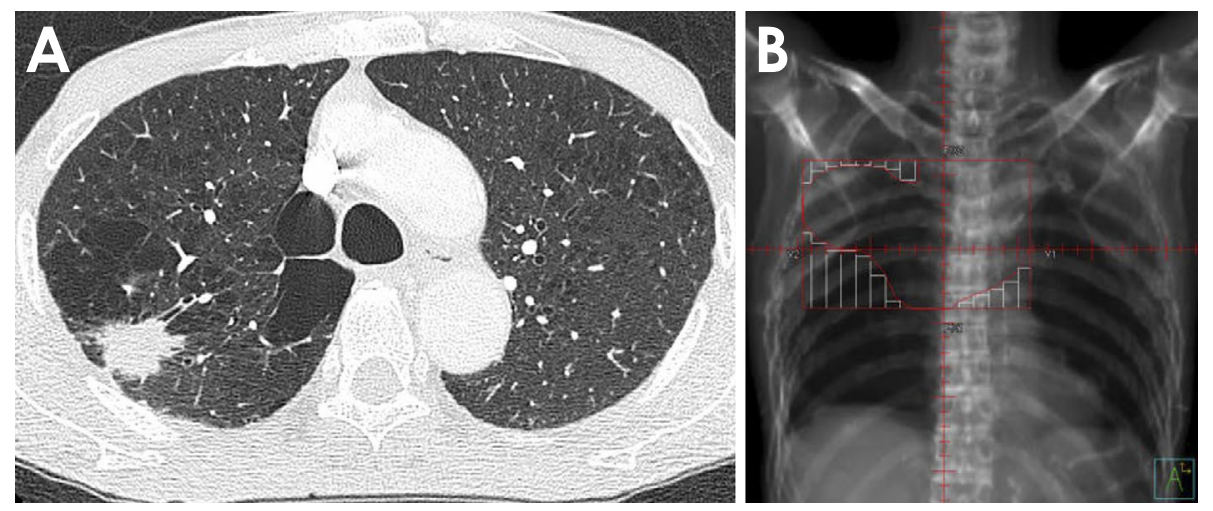

Figure 1. Lung cancer and irradiation field. (A) The primary lesion of the non-small cell lung cancer (NSCLC) of the right upper lobe on CT before concurrent chemoradiotherapy (CCRT). (B) Irradiation field (digitally reconstructed radiograph). The heart was outside of the irradiation field.
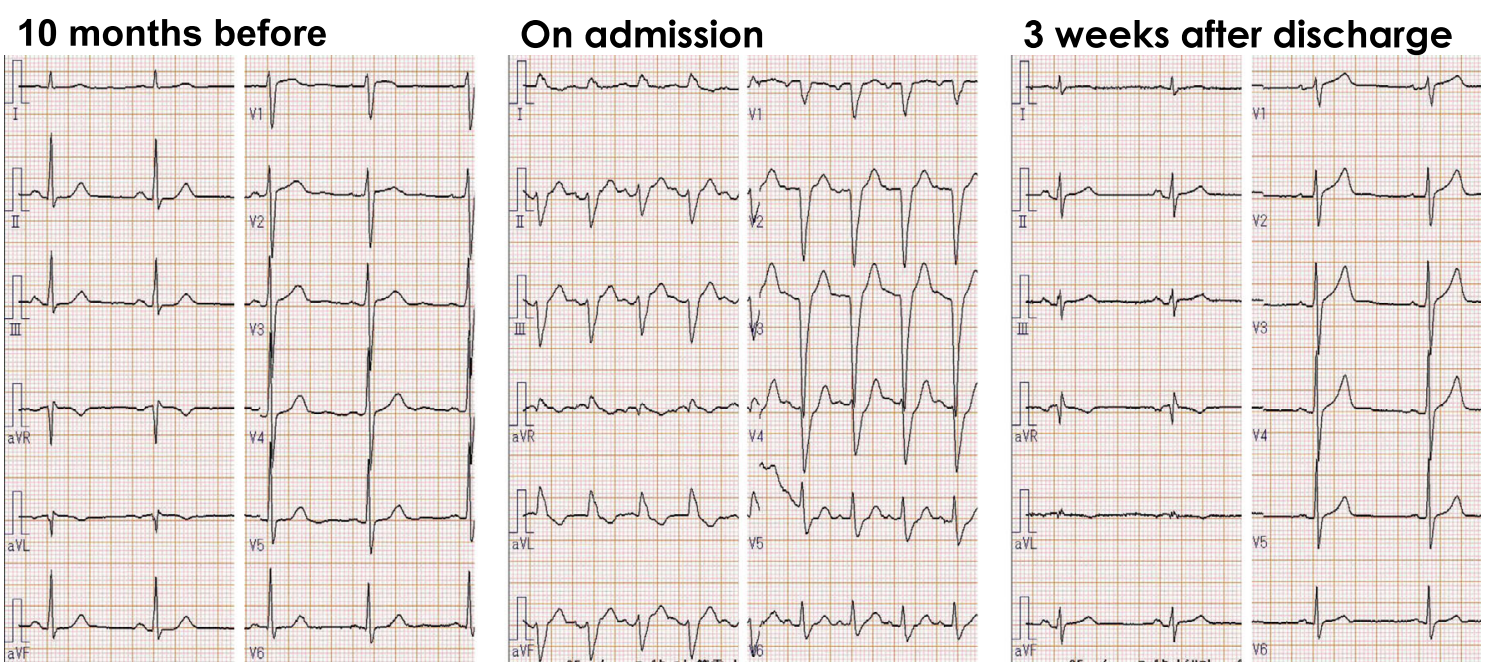

Figure 2. ECG changes. On admission, an ECG showing sinus rhythm, heart rate of $113 \mathrm{bpm}$, and the presence of complete left bundle bunch block (CLBBB), which was not seen 10 months earlier. CLBBB disappeared in a week after corticosteroid therapy. The ECG obtained three weeks after discharge is also shown in this Figure.

surgery. His daily medications were lansoprazole $15 \mathrm{mg}$, bisoprolol fumarate $2.5 \mathrm{mg}$, and telmisartan $20 \mathrm{mg}$.

On an examination, he was ill-appearing, with a lowgrade fever of $37.3^{\circ} \mathrm{C}$. He had a blood pressure of $111 / 87$ $\mathrm{mmHg}$, regular pulse of 95 beats/min, and respiratory rate of 40 breaths $/ \mathrm{min}$ with $\mathrm{O}_{2}$ saturation of $87 \%$ on room air. Heart sounds were normal. His chest sounds showed bilateral late-inspiratory crackles. He did not have leg edema or muscle pain. Laboratory data showed that the white blood cell count was $6,200 / \mathrm{mm}^{3}$, hemoglobin level was $13.2 \mathrm{~g} / \mathrm{dL}$, platelet count was $243,000 / \mathrm{mm}^{3}$, elevated creatine kinase (CK) level was $326 \mathrm{IU} / \mathrm{L}$, troponin I level was $14.9 \mathrm{ng} / \mathrm{mL}$, and brain natriuretic peptide (BNP) level was $841.8 \mathrm{pg} / \mathrm{ml}$. The C-reactive protein (CRP) value increased to $3.24 \mathrm{mg}$ / $\mathrm{dL}$. The results of autoantibody tests, including antinuclear antibodies, antineutrophil cytoplasmic antibodies (ANCA), anti-ARS antibodies, anti-MDA-5 antibodies, anti-Mi-2 antibodies, and anti-transcription intermediary factor (TIF)1-g antibodies, were negative. An Enterovirus genus RNA test (including Coxsackievirus, Poliovirus, and Echovirus) of his serum was also negative.

An electrocardiogram (ECG) showed new left bundle branch block (LBBB) (Fig. 2). Echocardiography revealed diffuse hypokinesis of the wall motion (especially inferior wall), and his ejection fraction was $30 \%$. However, the abnormal wall motion area did not follow the distribution of the coronary artery. This finding was inconsistent with coronary artery disease. Chest X-ray showed cardiomegaly and enhancement in the pulmonary vascular shadow (Fig. 3A). Chest computed tomography (CT) revealed bilateral diffuse ground-glass opacity, pleural effusion, and pericardial effusion (Fig. 3B). Cardiac magnetic resonance imaging (CMRI) showed a high signal intensity of the inferior wall of the base on T2-weighted imaging black blood (T2WI-BB) (Fig. 4A). Signal enhancement was also seen on late gadolinium enhancement (LGE) imaging of the anteroseptal and anterior free wall of the apex and posterior wall of the base (Fig. 4B). He underwent coronary angiography, which re- 


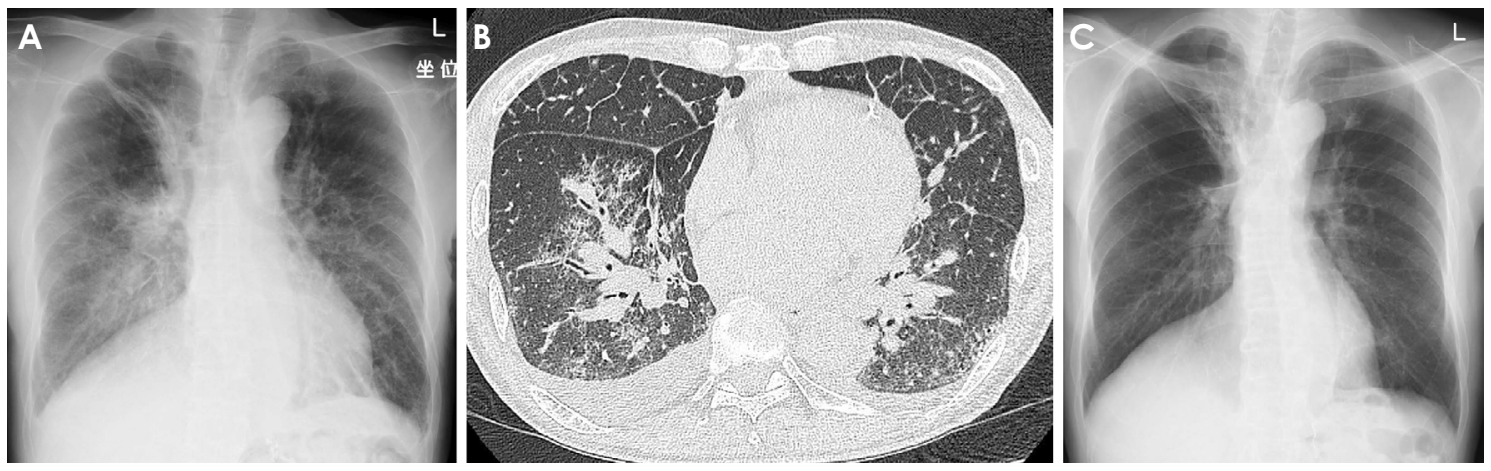

Figure 3. Chest X-ray and CT. (A) Cardiomegaly and vascular shadow enhancement on Chest Xray on admission. (B) Chest CT showing bilateral diffuse ground-glass opacity, pleural effusion, and pericardial effusion. (C) Chest X-ray on day 9 after admission. Abnormal findings on chest X-ray disappeared after a week, and chest X-ray and CT findings have continued to be stable for two years since the discharge.
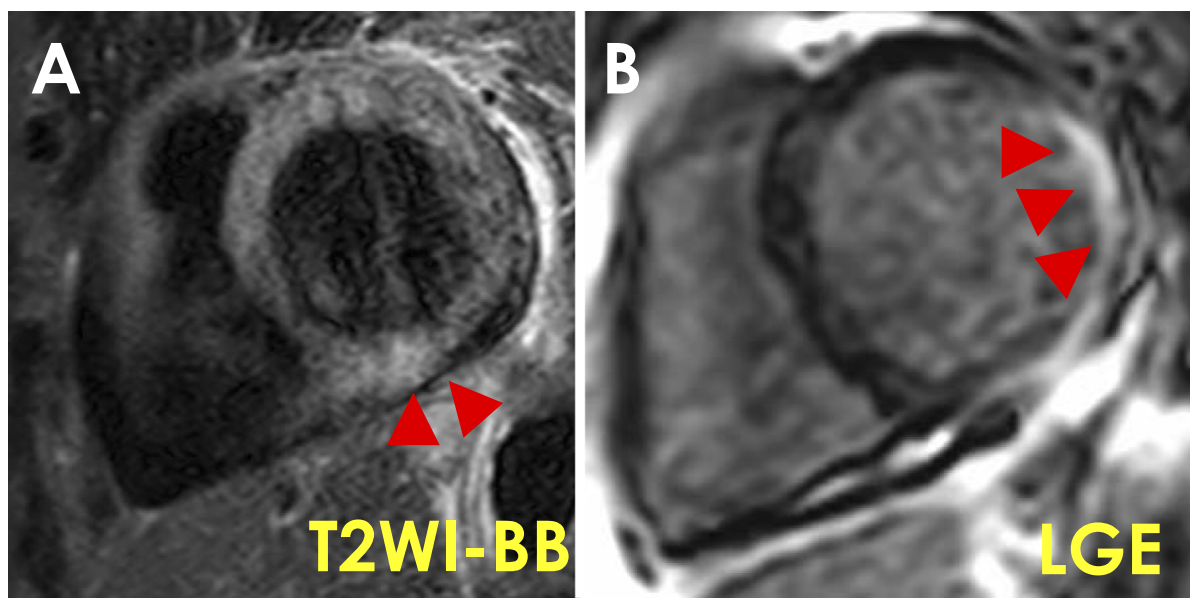

Figure 4. Cardiac MRI. (A) Inferior wall of the base (arrowheads) showing a high signal intensity on T2-weighted imaging black blood (T2WI-BB); (B) anteroseptal and anterior free wall of the apex, and posterior wall of the base (arrowheads) showing signal enhancement on late gadolinium enhancement (LGE) imaging.

vealed $75 \%$ stenosis of left anterior descending artery. Pulmonary artery catheterization showed his mean pulmonary capillary wedge pressure to be $10 \mathrm{mmHg}$ and his cardiac index to be $2.46 \mathrm{~L} / \mathrm{min} / \mathrm{m}^{2}$. A myocardial biopsy of the left ventricle was also performed, which revealed lymphocyte infiltration. Degeneration and fibrosis of myocardial cells were also found (Fig. 5).

He was suspected of having myocarditis due to an irAE because of the long administration of ICI for seven months and the findings of echocardiography, CMRI, and the myocardial biopsy. Although functional ischemia also existed, ischemia from coronary stenosis did not seem to be the main cause of myocardial damage, as coronary stenosis did not explain the hypokinesis of the wall motion. Bi-weekly administration of durvalumab was suspended, and he received intravenous methylprednisolone of $1,000 \mathrm{mg}$ for 3 days, followed by oral prednisolone of $60 \mathrm{mg}$. He was also treated with carperitide and non-invasive positive pressure ventilation for acute heart failure and respiratory failure. His oxygenation, chest radiography findings, and CLBBB on his ECG improved after a week. His CK and troponin I declined gradually. Oral prednisolone was gradually tapered, and he was discharged from the hospital on day 16 after admission. After discharge, prednisolone was continuously tapered.

The ejection fraction on echocardiography improved to $57 \%$ at 11 months after discharge. The findings of chest $\mathrm{X}$ ray and the ECG have also been stable (Fig. 2, 3C). The administration of durvalumab has been discontinued, and myocarditis has not recurred, even after the cessation of prednisolone. The lung cancer also continued to be stable for two years.

\section{Discussion}

We herein report a case of durvalumab-associated myocarditis that developed seven months after the first administration. Myocarditis was alleviated by corticosteroid therapy 


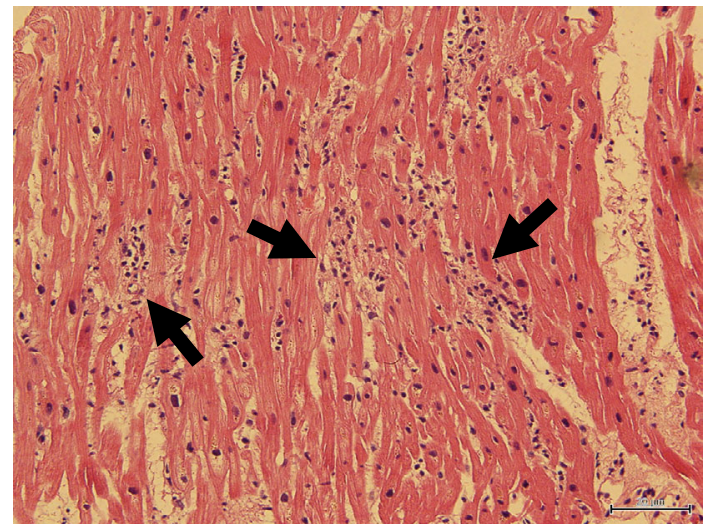

Figure 5. Myocardial biopsy findings. A myocardial biopsy showing lymphocyte infiltration (arrows) and degeneration and fibrosis of myocardial cells.

and did not recur after completing the administration of corticosteroids.

The symptoms of myocarditis are highly variable and can range from subclinical disease to fatigue, chest pain, heart failure, cardiogenic shock, arrhythmia, and sudden death (7-9). Combinations of clinical, biochemical, and imaging findings are helpful for the diagnosis of myocarditis. Furthermore, CMRI is playing an increasing role in the diagnosis of myocarditis. There are recommendations concerning the use of CMRI for the diagnosis of myocarditis, and diagnostic CMRI criteria for myocarditis have also been proposed (10). The present case met these criteria, with clinically suspected myocarditis, regional myocardial signal increase on T2-weighted imaging, and focal lesion with nonischemic regional distribution on LGE imaging. At present, an endomyocardial biopsy (EMB) is the gold standard for the diagnosis of myocarditis. A definitive diagnosis of myocarditis is established based on an EMB (Dallas criteria) (11). This case was histologically diagnosed as active myocarditis, based on inflammatory infiltration with degeneration of myocytes.

Infectious disease accounts for the etiology of most myocarditis cases in previously healthy patients, typically because of a direct viral infection or post-viral immunemediated reaction. Myocardial inflammation, however, may also be triggered by toxic, ischemic, or mechanical injury; drug-related inflammation; transplant rejection; or other immune reactions (10). Since myocarditis occurred after the long-term administration of ICIs and an Enterovirus genus RNA test of the blood was negative in this case, we suspected this case of myocarditis was highly associated with durvalumab. However, we were unable to completely exclude the possibility that this myocarditis had been induced by other causes, including minor viral infections, since myocarditis from irAEs was clinically and histologically indistinguishable from myocarditis induced by viral infections. The histological findings of myocarditis induced by irAEs were reported to be nonspecific T-cell-predominant lymphocytic infiltrate within the myocardium $(5,12)$. Therefore, it is generally difficult to confirm a clear causal relationship between ICIs and myocarditis, as with cases of other irAEs. However, as steroids are usually not used for viral myocarditis, steroid responsiveness may be a clue for the differentiation between the two, but further research is needed to state its validity (11).

Myocarditis induced by irAEs has not occurred frequently and is reported to account for approximately $1.14 \%$ of all irAEs (5). Several case series and case reports have described myocarditis related to ICIs, such as nivolumab, pembrolizumab, ipilimumab, tremelimumab, atezolizumab, avelumab, and durvalumab (3-5). Regarding durvalumab, there exists a case report of myocarditis occurring approximately four weeks after tremelimumab plus durvalumab treatment for endometrial cancer (6), but no cases of lung cancer with concomitant myocarditis associated with durvalumab have been reported.

In most of the reported cases, myocarditis occurred shortly after the first or second administration of ICIs. Patients who developed myocarditis in the earlier periods tend to have a more serious disease. Most of the patients who died were those who developed myocarditis in the early phases (13). Furthermore, in a case series of 35 patients, the median time to the onset of myocarditis from the first ICI administration was 34 days, with $81 \%$ presenting within 3 months of starting therapy (5). The present case is notable because its time to the onset was seven months after ICI administration. Some cases of myocarditis that occurred over six months after starting ICIs have also been reported (5), similar to our case. Physicians must recognize that irAEs can occur at any phase.

Generally, immunosuppressive therapy including corticosteroids is considered in non-viral myocarditis, especially autoimmune myocarditis (11). There is, to our knowledge, no evidence or case report describing the harmful effects of corticosteroids on myocarditis, including viral myocarditis. Furthermore, the benefits of corticosteroids for treating viral myocarditis of COVID-19 have recently been mentioned (14). Thus, when autoimmune etiology is suspected and the severity of the patient's state is critical, we may administer corticosteroids without hesitation, even if the cause of myocarditis is not completely determined.

Guidelines for irAEs suggest that, in the presence of severe myocarditis related to ICIs, immunotherapy should be permanently discontinued, and high doses of steroids (prednisolone 1-2 $\mathrm{mg} / \mathrm{kg}$ ) should be considered (15). In one study, with a high starting dose of immunosuppression, the serum maximum troponin was low, and there was a low rate of major adverse cardiovascular events (MACEs). The final/ discharge serum troponin level was also low among those treated with high initial doses of steroids (5). In addition, corticosteroid use was reported to be associated with a high probability of left ventricular function recovery (16).

Treatment of myocarditis includes general nonspecific measures to treat the heart disease, including heart failure therapy, such as diuretics, angiotensin-converting enzyme in- 
hibitor, or angiotensin receptor blockade, and betaadrenergic blockade (11). Steroid pulse therapy followed by high doses of prednisolone along with medications for heart failure seemed to be effective in the treatment of myocarditis in this case. However, it is reported that MACEs still occurred in several cases with an initial dose of methylprednisolone of $1,000 \mathrm{mg}$ (5). Therefore, we should monitor the condition of the patient carefully even after the administration of corticosteroids.

The present patient's lung cancer has not recurred for two years, although he has not been treated with anticancer drugs, including ICIs. Generally, the occurrence of irAEs is associated with a good clinical prognosis; indeed, several studies have reported more favorable outcomes in patients with NSCLC who developed irAEs than in those who did not (17-19). However, to our knowledge, there have been no previous studies on the relationship between ICI-associated myocarditis and the clinical effectiveness of ICIs. This may be because myocarditis associated with ICIs is rare. As in the present case, the development of myocarditis may be associated with a better tumor response, but further research is required to confirm this association.

\section{Conclusion}

In conclusion, we experienced a case of late-onset myocarditis following the administration of durvalumab, successfully treated with corticosteroids. Myocarditis may lead to life-threatening events, such as shock or death. Early recognition is important, as prompt treatment with corticosteroids and heart failure therapy can increase the chance of a survival. Myocarditis tends to happen in the early period of ICI administration but sometimes occurs several months after its initial treatment. Therefore, we should be aware of this disease and should check the ECG or presence of myocardial markers when patients using ICIs present with a series of symptoms related with heart, regardless of the length of treatment with ICIs.

The authors state that they have no Conflict of Interest (COI).

\section{References}

1. Antonia SJ, Villegas A, Daniel D, et al. Overall Survival with Durvalumab after Chemoradiotherapy in Stage III NSCLC. N Engl J Med 379: 2342-2350, 2018.

2. Brahmer JR, Lacchetti C, Schneider BJ, et al. Management of Immune-Related Adverse Events in Patients Treated With Immune Checkpoint Inhibitor Therapy: American Society of Clinical Oncology Clinical Practice Guideline. J Clin Oncol 36: 1714-1768, 2018.

3. Tan JL, Mugwagwa AN, Cieslik L, et al. Nivolumab-induced myocarditis complicated by complete atrioventricular block in a patient with metastatic non-small cell lung cancer. BMJ Case Rep 12: e229963, 2019.

4. Matsuo K, Ishiguro T, Najama T, et al. Nivolumab-induced Myocarditis Successfully Treated with Corticosteroid Therapy: A Case Report and Review of the Literature. Intern Med 58: 2367-2372, 2019.

5. Mahmood SS, Fradley MG, Cohen JV. Myocarditis in Patients Treated With Immune Checkpoint Inhibitors. J Am Coll Cardiol 71: 1755-1176, 2018.

6. Mahmood SS, Chen CL, Shapnik N, et al. Myocarditis with tremelimumab plus durvalumab combination therapy for endometrial cancer: A case report. Gynecol Oncol Rep 25: 74-77, 2018.

7. Cooper LT Jr. Myocarditis. N Engl J Med 360: 1526-1538, 2009.

8. Blauwet LA, Cooper LT. Myocarditis. Prog Cardiovasc Dis 52: 274-288, 2010.

9. Caforio AL, Calabrese F, Angelini A, et al. A prospective study of biopsy-proven myocarditis: prognostic relevance of clinical and aetiopathogenetic features at diagnosis. Eur Heart J 28: 1326-1333, 2007.

10. Friedrich MG, Sechtem U, Schulz-Menger J, et al. Cardiovascular magnetic resonance in myocarditis: A JACC White Paper. J Am Coll Cardiol 53: 1475-1487, 2009.

11. Caforio AL, Pankuweit S, Arbustini E, et al. Current state of knowledge on aetiology, diagnosis, management, and therapy of myocarditis: a position statement of the European Society of Cardiology Working Group on Myocardial and Pericardial Diseases. Eur Heart J 34: 2636-2648, 2013.

12. Moslehi J, Lichtman AH, Sharpe AH, et al. Immune checkpoint inhibitor-associated myocarditis: manifestations and mechanisms. J Clin Invest 131: e145186, 2021.

13. Atallah-Yunes SA, Kadado AJ, Kaufman GP, et al. Immune checkpoint inhibitor therapy and myocarditis: a systematic review of reported cases. J Cancer Res Clin Oncol 145: 1527-1557, 2019.

14. Kamarullah W Nurcahyani, Mary Josephine C, et al. Corticosteroid Therapy in Management of Myocarditis Associated with COVID-19; a Systematic Review of Current Evidence. Arch Acad Emerg Med 9: e32, 2021.

15. Thompson JA, Schneider BJ, Brahmer J, et al. Management of Immunotherapy-Related Toxicities, Version 1.2019. J Natl Compr Canc Netw 17: 255-289, 2019.

16. Escudier M, Cautela J, Malissen N, et al. Clinical Features, Management, and Outcomes of Immune Checkpoint Inhibitor-Related Cardiotoxicity. Circulation 136: 2085-2087, 2017.

17. Teraoka S, Fujimoto D, Morimoto T, et al. Early Immune-Related Adverse Events and Association with Outcome in Advanced NonSmall Cell Lung Cancer Patients Treated with Nivolumab: A Prospective Cohort Study. J Thorac Oncol 12: 1798-1805, 2017.

18. Sato K, Akamatsu H, Murakami E, et al. Correlation between immune-related adverse events and efficacy in non-small cell lung cancer treated with nivolumab. Lung Cancer 115: 71-74, 2018.

19. Toi Y, Sugawara S, Kawashima Y, et al. Association of ImmuneRelated Adverse Events with Clinical Benefit in Patients with Advanced Non-Small-Cell Lung Cancer Treated with Nivolumab. Oncologist 23: 1358-1365, 2018.

The Internal Medicine is an Open Access journal distributed under the Creative Commons Attribution-NonCommercial-NoDerivatives 4.0 International License. To view the details of this license, please visit (https://creativecommons.org/licenses/ by-nc-nd/4.0/).

(C) The Japanese Society of Internal Medicine Intern Med Advance Publication 